NATHALIE SINCLAIR, RINA ZAZKIS and PETER LILJEDAHL

\title{
NUMBER WORLDS: VISUAL AND EXPERIMENTAL ACCESS TO ELEMENTARY NUMBER THEORY CONCEPTS
}

\begin{abstract}
Recent research demonstrates that many issues related to the structure of natural numbers and the relationship among numbers are not well grasped by students. In this article, we describe a computer-based learning environment called Number Worlds that was designed to support the exploration of elementary number theory concepts by making the essential relationships and patterns more accessible to learners. Based on our research with pre-service elementary school teachers, we show how both the visual representations embedded in the microworld, and the possibilities afforded for experimentation affect learners' understanding and appreciation of basic concepts in elementary number theory. We also discuss the aesthetic and affective dimensions of the research participants' engagement with the learning environment.
\end{abstract}

KEY WORDS: elementary number theory, experimentation, microworlds, visualisation

\section{INTRODUCTION}

Despite the fact that many professional mathematicians see number theory as the 'queen of mathematics,' and the locus of the most elegant, fundamental and powerful ideas in mathematics, pre-service elementary school teachers frequently fail to gain access to its most basic structures and relationships. Yet, as the NCTM Standards (1989) state, number theory explorations "have payoffs in problem solving, in understanding and developing mathematical concepts, in illustrating the beauty of mathematics, and in understanding the human aspects of the historical development of number" (p. 91). In this study, we engaged pre-service elementary school teachers in a novel, computer-based learning environment that offered possibilities for experimental approaches and productive visualisations. One goal of the study was to determine whether such explorations could affect participants' understanding of elementary number theory structures and relationships. A second goal was to use the computer-based learning environment as a 'window' on mathematical meaning-making in order to better understand the intuitions, perceptions and difficulties that learners have with concepts related to the natural numbers.

We begin the paper by discussing the difficulties encountered by learners when working with elementary number theory, as identified in previous research. Next, we outline the motivations for and the design prin- 
ciples used in the development of a computer-based learning environment called Number Worlds, which aims to address and overcome some of these difficulties. We then describe the learning environment and outline some of its mathematical features. Finally, we report on a study we conducted with a large group of pre-service elementary teachers who used Number Worlds to explore several ideas in elementary number theory.

\section{SUMMARY OF RESEARCH IN ELEMENTARY NUMBER THEORY}

Current trends in mathematics education, at all age levels, reflect a primary concern with utility and the applicability of mathematical content. Thus, frequently, mathematical structure and relationships are overlooked in teaching. It is not surprising then, that recent research demonstrates how many issues related to the structure of natural numbers and the relationship among numbers are not well grasped by learners. We summarise here several examples relevant to our study.

- Onfactors and multiples. Prior research demonstrates that concepts of factors and multiples present a challenge for pre-service elementary school teachers. It has been noted that learners often confuse and interchange these terms (Zazkis, 2000) and often do not grasp the connection between factors and multiples. That is to say, the implication ' $a$ is a factor of $b$ ' if and only if ' $b$ is a multiple of $a$ ' is not utilised in problem situations (Zazkis, 2000; Brown, Thomas and Tolias, 2002).

- On factors. Prior research recognises several beliefs related to factors of a number that are not uncommon among pre-service teachers. In Zazkis (1999), some research participants expressed such beliefs explicitly, while for others they were 'beliefs in action', observed through their approaches to posed problems. A number of participants claimed that large numbers have more factors than small numbers. When reminded of the existence of 'very large' prime numbers, this belief was refined to claim that large composite numbers have more factors than small composite numbers. In other research, Zazkis and Campbell (1996b) noted that participants believed that a large composite number must have a small prime factor. Following this belief some participants concluded that $391(391=17 \times 23)$ is a prime number because it is not divisible by primes below 10 . This finding has been reconfirmed also with larger numbers, when a few participants in a study by Zazkis and Liljedahl (under review) checked divisibility by a few 'small' primes and concluded that a number F, 
given as $\mathrm{F}=151 \times 157$ is prime. A more striking finding of this latest study is that one half of the participants did not immediately recognise a number represented as a product as being a composite number.

- On multiples. Mathematicians, and those who are generally mathematically inclined, often take for granted the property that every $n$th number is a multiple of $n$. Yet research shows that for many learners this property is not obvious. Pre-service teachers in Zazkis and Campbell's (1996a) study often performed long division in order to confirm that there is indeed a number divisible by 7 in a given interval of 10 numbers. Moreover, even engineering and computer science majors had difficulty in determining whether there is a number $x$ divisible by 8 , such that $\mathrm{M} \leq x \leq \mathrm{M}+10$, for a given $\mathrm{M}$ (Ferrari, 2002). Zazkis and Liljedahl (2002) addressed understanding of the same property in their investigation of arithmetic sequences. Presented with the first few terms of an infinite arithmetic sequence, participants were asked to determine whether a certain 'large' number is an element of the sequence. Participants approached such problems by first distinguishing between sequences of multiples (e.g., 7, 14, 21, ..), which they perceived as "orderly", and sequences of non-multiples, (e.g., 8, $15,22, \ldots)$, which they perceived as "sporadic". Analysis indicated that significant progress in understanding occurs when one recognises arithmetic sequences of 'non-multiples' as 'multiples shifted'.

\section{A COMPUTER-BASED APPROACH TO NUMBER THEORY LEARNING}

To satisfy both our research aims - to better understand some of the learning difficulties summarised above - and our teaching objectives to provide students with more powerful ways of learning - one author (Sinclair) designed a computer-based learning environment focusing on the domain of elementary number theory. We first outline our rationale for developing this environment, a number theory microworld. Then we describe the design principles we followed, and their connection to the research summarised above. Finally, we present an analysis of the mathematical concepts and behaviours supported by the microworld.

\section{Affordances of Computer-based Learning Environments}

Many researchers, such as Goldenberg (1989), have argued that welldesigned computer-based learning environments can provide a scaffold for reasoning by fostering the development and use of visual and experimental reasoning styles, which greatly complement the traditionally-taught 
symbolic-deductive methods. For the learning of elementary number theory, few, if any, such computer-based environments exist. In addition, little is known about the kinds of visual representations and experimental interfaces that might help students learn and understand the wide range of number theory-related ideas involved in the research summarised above. Certain mathematical subjects, for example fractal geometry, easily lend themselves to a visual and experimental form of mathematical inquiry, but is the same necessarily true of number theory? We were aware that some professional number theorists take advantage of the visual and experimental approaches afforded by the computer; however, we wanted to establish whether such approaches are possible and appropriate for student learning.

Factor trees and number lines provide one type of visual representation frequently encountered in the sub-domain of elementary number theory. Alongside such visual tools, push-button calculators can support a certain range of numeric experimentation. However, these tools suffer from a degree of isolation: for example, neither factor trees nor number lines can be represented on calculators. In contrast to an approach based on collections of tools, we were interested in the class of learning environments called "microworlds" which, as Edwards (1995) describes, "embody" or "instantiate" some sub-domain of mathematics. The microworld is intended to be a mini-domain of mathematics that offers an external representation of a (sub)set of mathematical ideas, and brings tools together into a phenomenological whole. The challenge for mathematics educators is to design microworlds that can offer new external systems of representation that foster more effective learning and problem solving.

Noss and Hoyles (1996) argue that the computational objects of a microworld - its basic building blocks - should maximise the chance to forge links with mathematical objects and relationships. In the case of elementary number theory, the computational objects should maximise the learner's chance to explore relationships among numbers, as well as their underlying structure. Noss and Hoyles also argue that the development of a microworld should involve predicting where student 'breakdowns' might occur: breakdowns are incidents where learners' anticipated outcomes are not experienced.

One final design issue we wanted to bear in mind relates to the difficulties involved in incorporating visualisation into mathematical activity. We recognised that without proper support, students may be either unwilling or unable to take advantage of potentially powerful visualisations (see Eisenberg and Dreyfus, 1991). We also recognised that microworlds are essentially inert without the animating presence of both the student and the task that invites investigation. The design of our 
learning environment thus included as well the creation of a set of accompanying tasks that would encourage connections between analytic and visual modes of reasoning (see Appendix 1). Many of these tasks involved the comparison or simultaneous use of factors and multiples, while others were intended to target particular properties of factors and multiples.

\section{Description of the Number Worlds Microworld}

Figure 1 shows a snapshot of the Number Worlds applet. ${ }^{1}$ The centre grid contains a two-dimensional array of clickable cells. The numbers shown in the cells depend on the 'world' that has been chosen. The basic objects of Number Worlds are the positive integers, which we call the Natural World, but the user can choose among other sets: the Whole World (i.e., the positive integers and the number 0), Even World, Odd World, and Prime World. ${ }^{2}$ Each world displays the set of numbers involved.

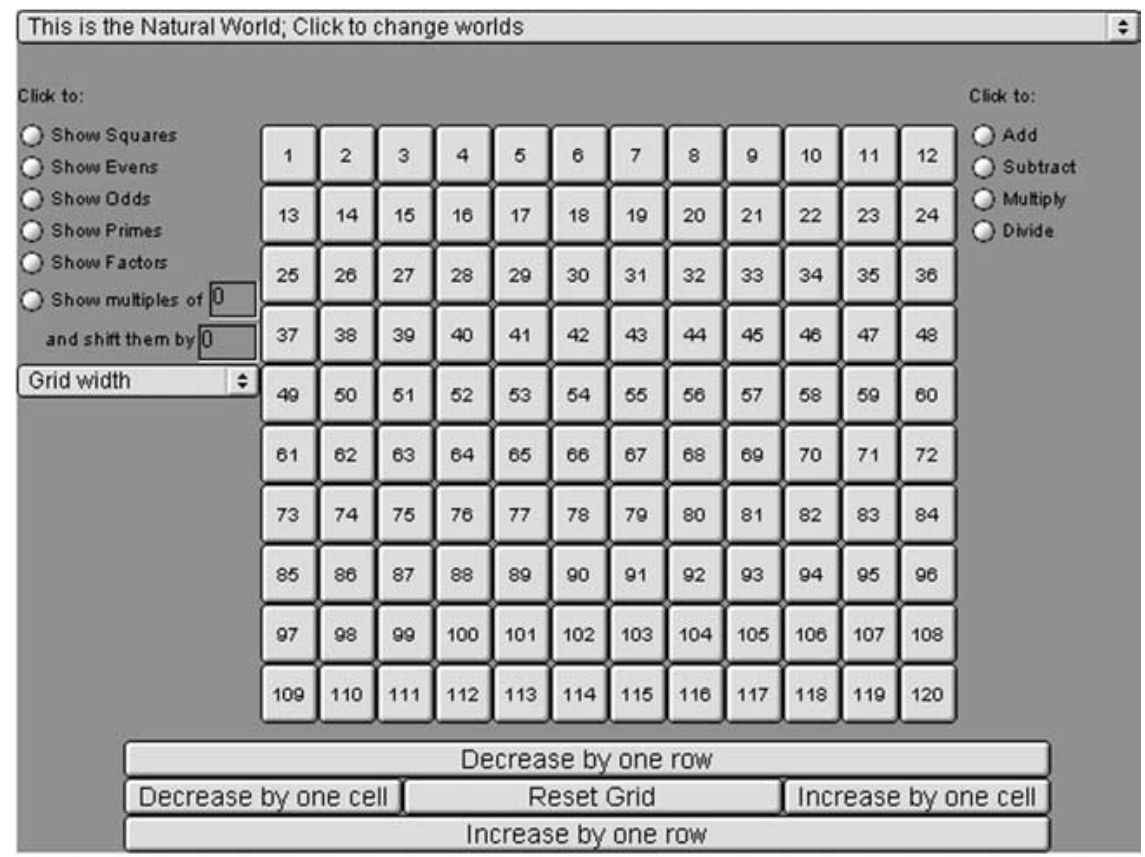

Figure 1. The Number Worlds microworld.

It is also possible to change the numbers shown in the cells. The user can increase or decrease the start number by 'one row', that is, by the value of the grid width, or simply by one 'cell'. The Reset Grid button resets the start number to the first number of the current world.

The appearance of the grid can be affected by changing the value of the Grid width menu. By selecting values from one to twelve, the user 
can change the number of columns displayed, and thus the total number of cells. However there are always ten rows.

Within each world, the user can choose to highlight certain types of numbers: Squares, Evens, Odds, Primes, Factors, and Multiples. Further, the multiples that are chosen can be shifted by an integer in order to create any arithmetic sequence.

Finally in the applet, the four basic arithmetic operations are available to the user. The result of the operation, in addition to the two inputs, is highlighted on the grid while a text display of the operation and the result appears above the grid.

\section{Mathematical Ideas Inherent in Number Worlds}

Elementary number theory is concerned with the structures and relationships of natural numbers. Therefore, in designing the Number Worlds microworld, we have chosen to focus primarily on this set of numbers. However, instead of the one-dimensional number line representation that is traditionally used, we adopted a two-dimensional grid display, thus maximising the use of 'real estate' on the screen. The two-dimensional grid display also sheds a different light on the relationships among the numbers and provides an opportunity to construct or reconstruct these relationships. In addition, it offers a different external representation of both factors and multiples, as well as primes and square numbers, in ways that provide concrete visual instantiations of algebraic relationships. Further, by producing unexpected patterns, the two-dimensional grid display offers a novel representation of the numbers, which we hoped would provoke surprise and engagement for learners. In what follows we provide several examples of the mathematical relationships inherent in Number Worlds.

\section{Example 1: Stripes and Divisibility}

The relationship between the input 'show multiples of' and the grid width determines the type of pattern. For example, in the Natural World, showing multiples of ten on a grid width of ten results in one highlighted column (see Figure 2) referred to as a 'stripe'. Showing multiples of five or two on the grid width of ten results in two or five highlighted stripes, respectively. In general, a pattern of stripes emerges when the grid width is divisible by the input to 'show multiples of'.

\section{Example 2: Stripes Shifted and What 'Counts' as the First Multiple?}

Showing multiples of ten on a grid width of ten forms a stripe; this stripe is 'shifted' when the multiples are shifted. However, in order to have all the numbers in the stripe highlighted, a negative shift is required; the shifts of adding two and subtracting eight in the Natural World are shown in 


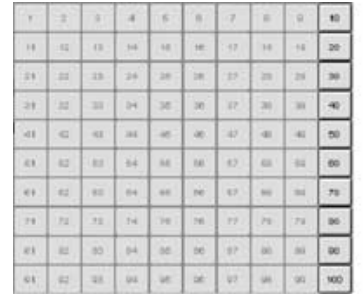

(a) Multiples of $10, \mathrm{gw}=10$

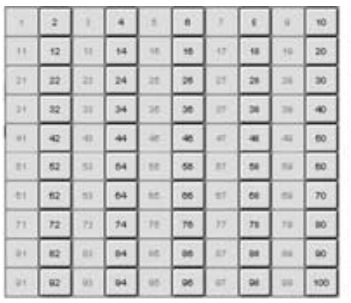

(b) Multiples of 2, gw $=10$

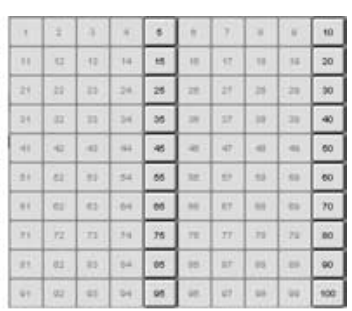

(c) Multiples of 5, gw $=10$

Figure 2. Showing multiples on a grid width of ten.

Figure 3. Of course, when working in the Whole World, which includes zero, this is no longer the case. Showing the multiples of ten, shift two would highlight the numbers $2,12,22,32$, etc.

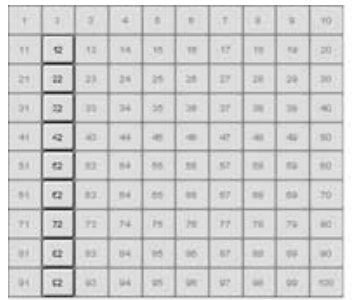

(a) Multiples of 10, shift 2

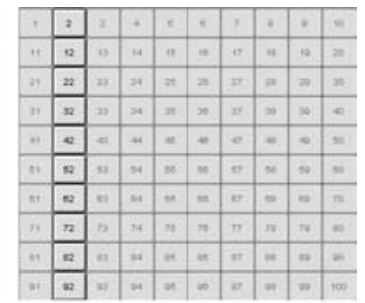

(b) Multiples of 10 , shift -8

Figure 3. Using the 'shift' functionality.

\section{Example 3: Diagonals and Non-divisibility}

As shown above, a stripe pattern emerges when the input to multiples $m$ is a factor of the grid width $n$. However, this is not the only way to produce a recognisable pattern. Figure 4 shows multiples of 2, 4, 5, and 7 on a grid width of 9. We refer to the patterns in (b) and (c) as 'diagonals', and refer to the pattern in (d) as 'disconnected diagonals'. The pattern in (a) can be seen as diagonals as well; however, it is more naturally identified as a checkerboard pattern.

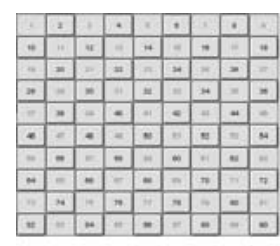

(a) $\mathrm{m}=2, \mathrm{gw}=9$

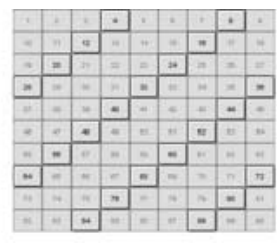

(b) $\mathrm{m}=4$, gw $=9$

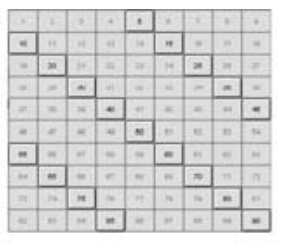

(c) $\mathrm{m}=5$, gw $=9$

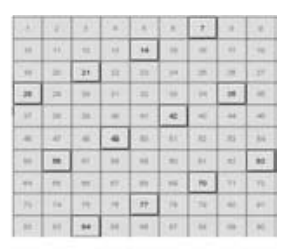

(d) $\mathrm{m}=7, \mathrm{gw}=9$

Figure 4. Relationships between multiples and grid widths. 
These images are examples of the following general relationship: 'diagonals' are displayed by multiples of $m$ on a grid width of $n$, where $n \pm 1$ is divisible by $m$, and 'disconnected diagonals' are displayed by multiples of $m$ on a grid width of $n$, where neither $n$ nor $n \pm 1$ are divisible by $m$. 'Checkerboards' are a special case of 'diagonals' and they are displayed by multiples of two on any odd grid width. (So in fact this precludes the creation of a 'real' checkerboard pattern of width eight.)

By stacking the common differences on top of each other, the 'common-ness' of the differences becomes visually obvious even when diagonals, rather than columns, are being displayed. We attend to this feature below, in our description of the research participants' work with the microworld.

\section{Example 4: Shift and Congruence Classes}

In examples 2 and 3, we described how the relationships between the grid width and the input to 'show multiples of' affect the visual patterns created on the grid. These patterns hold for any shift value; increasing the value of the shift will simply 'move' the pattern over. To illustrate this, Figure 5 invites the reader to compare multiples of 5 , shift 0 - as set by default and multiples of 5 , shift 3 .

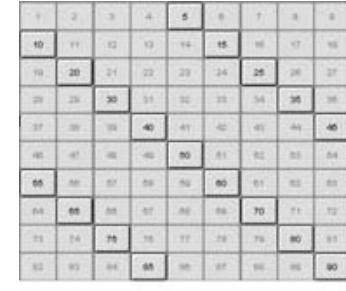

(a) $\mathrm{m}=5, \mathrm{~s}=0$

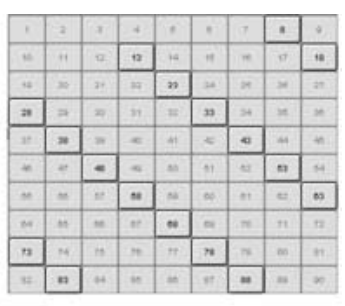

(b) $\mathrm{m}=5, \mathrm{~s}=3$

Figure 5. Illustrating the effect of the shift on the pattern.

In general, by showing multiples with a shift, the user can generate any arithmetic sequence. The input for 'show multiples of' corresponds to the common difference while the initial term is the sum of the inputs to 'show multiples' and 'shift by' in the Natural World. From a different mathematical point of view, the set of numbers highlighted by showing multiples of $m$ with a shift of $s$ actually forms a congruence class modulo $s$ (using the convention that, for integers $a$ and $b, a$ is congruent to $b$ modulo $m$ if and only if $m$ divides $a-b$; in other words, $a$ and $b$ have the same remainder in division by $m$ ). 
Example 5: Prime Numbers

When prime numbers are highlighted on a grid of width 10 in the Natural World, one can see that the fourth, sixth, eighth and tenth columns are 'empty' - devoid of prime numbers. No highlighted cells appear in these columns since they contain only even numbers, which are divisible by 2 and therefore not prime. In addition, the fifth column contains only multiples of 5 (since the grid width is 10) and is thus similarly 'empty', except for the first cell. If the user selects Show Primes within the Odd World, the cells with the familiar prime numbers will be highlighted; however, all twin primes will appear in adjacent cells. Further, if the user selects Show Odds in the Prime World, every cell but the first will be highlighted (every prime number, except for 2 , is odd).

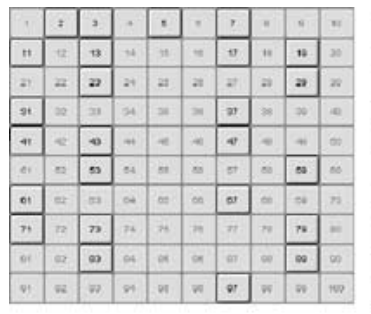

(a) Primes in Natural World

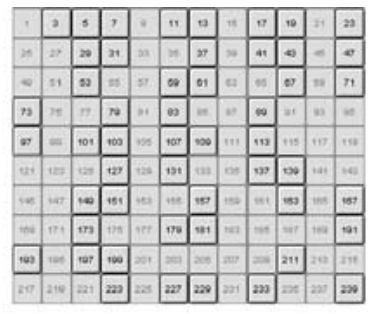

(b) Primes in Odd World

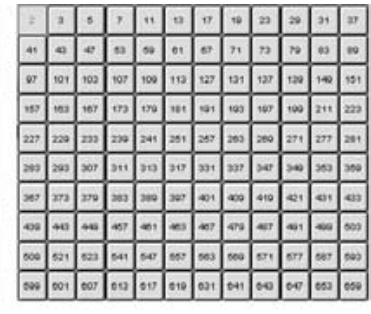

(c) Odds in Prime World

Figure 6. Highlighting cells in different worlds.

\section{Example 6: Arithmetic Operations and Closure}

The operations of addition, subtraction, and multiplication function as they do on a regular calculator. However, the division operation returns the (whole number) quotient and the remainder, in order to preserve consistency with the number theory theme of the microworld. The arithmetic operations were included primarily for investigation of closure properties. If the result of an operation does not belong to the chosen number world, a message saying so appears above the grid. For example, the result of the operation 5-17 does not belong to the Natural World; the result of the operation $5 \times 11$ does not belong to the Prime World; the result of operation $3+9$ does not belong to the Odd World.

\section{LEARNING WITH NUMBER WORLDS}

We asked 90 pre-service elementary school teachers, enrolled in a course Principles of Mathematics for Teachers, to use Number Worlds. The course includes a chapter on elementary number theory that deals in part with 
concepts of divisibility, divisibility rules, prime and composite numbers, factors and multiples, greatest common factor and least common multiple. This chapter had been completed by the teachers shortly before they were introduced to Number Worlds.

The participants were provided with written instructions about web access to Number Worlds, a description of the microworld's commands and capabilities, and a list of suggestions for explorations to be carried out before turning to the main assignment. The list of tasks for exploration is found in Appendix 1; two examples of these are:

- Can you find a number smaller than 45 that has more factors than the number 45 ?

- Can you get the numbers $7,14,21, \ldots$ highlighted? Can you get the numbers $8,15,22, \ldots$ highlighted?

These tasks were intended to familiarise the participants with all the aspects of Number Worlds and to create an environment of experimentation and conjecturing. To assist the participants, computer labs were scheduled at different times on different days. Participation in the lab was optional; nevertheless about one-half of the participants chose to work, for at least part of the assignment, in this environment.

Our data consists of three main sources: observations of participants' work during the lab time, written assignments and clinical interviews:

1. During the allocated lab hours, the work of the participants was observed and assistance given where necessary. We noted their frequently asked questions, chosen routes for exploration, conjectures, as well as their approaches toward testing conjectures. We also used these observations as a guideline for designing the interview questions.

2. Following the tasks for exploration, participants were provided with an additional list of tasks, for which a written response was requested. Participants had two weeks to complete the assignment. The list of tasks can be found in Appendix 2. These tasks specifically request that the participants make an observation and, whenever possible, generalise it. For example: Describe at least three different ways for creating a grid of diagonals. Describe a general procedure for creating a grid of diagonals. Explanations were expected, even when not explicitly requested, as a routine practice in the course.

3. After the completion of the written assignment, clinical interviews were conducted with seventeen volunteers from the group. The interviews lasted 30-50 minutes; the Number Worlds microworld was available to the participants at all times during the interview. These interviews were semi-structured, that is, the questions were designed 
in advance (see Appendix 3), but the interviewer had the liberty to follow up with prompts, include additional questions, or omit questions due to time considerations.

The data from the three sources were analysed according to two main themes: (1) the effects of visualisation and (2) the effects of experimentation. In addressing both themes we used Number Worlds as a lens (window) on the research participants' understanding of elementary number theory concepts, in particular the concepts of factor and multiple. We also attempted to examine their emerging understanding with respect to several difficulties and obstacles reported in prior research.

\section{Effects of Visual Representations Available in Number Worlds}

In this section, we examine the effects of visual representation on the participants' understanding of several concepts. For clarity, we structure our discussion in terms of the following two categories: the general concept of multiples; and, the difference between multiples and factors. In both categories, the visual representations available in the microworld seemed to provide our research participants with a structural - as opposed to procedural - way of perceiving the concepts of factors and multiples.

\section{Understandings of Multiples}

The participants' representations of multiples seem to have been affected by Number World's visual display, as evidenced in both the interviews and assignments. In contrast to the way that multiples are typically presented, as a list (such as $4,8,12,16$, etc.) which draws attention to individual numbers, the Number Worlds display emphasises the space in between subsequent highlighted numbers - and this space is constant for multiples. The participants seemed to form an image of multiples as possessing the property of 'constant spread'. One of the interview questions (see Question 6, Appendix 3) consisted in asking students to identify four different images, representing primes, factors, square numbers, or multiples (see Figure 7). Every interviewed participant immediately recognised the image of multiples. Of the sixteen explanations we elicited, twelve participants appealed to the visually discernible spacing between consecutive highlighted cells. Andrew's reasoning is characteristic of these: "So that's multiples, because you have the same amount of space between each, I think it's multiples of 5".

The notion of spread may seem obvious to the mathematically inclined, but Andrew - one of the strongest students in the class - commented on how it was the microworld that helped draw his attention to it. "Until you see it" he explained, you don't know "there's always going to be the 

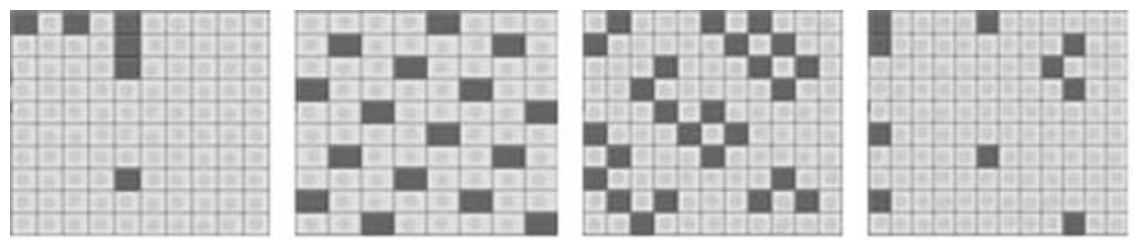

Figure 7. Four images taken from Number Worlds.

same amount of space". The notion of 'constant spread' was also prevalent in the assignment, and was used by the majority of the participants to describe their visual images of multiples. We also saw strong evidence of an if-and-only-if understanding of the 'constant spread' idea in several cases. Every interviewed participant was able to deduce that the three other images presented in the interview Question 6 (factors, square numbers, and primes) were not multiples because they did not have the same amount of space between highlighted numbers. Kate's observation was typical: "It doesn't have a pattern, like every 5th or whatever number".

The 'constant spread' feature of multiples is a visual perception of the property 'every nth number is a multiple of $n$ '. As mentioned earlier, this property - to which we refer as the 'every nth' property - was not capitalised upon by many participants in prior research studies (Zazkis and Campbell, 1996; Ferrari, 2002). In fact, interview Question 5 - 'Is there a multiple of 7 in the 9 th row of your grid? What about the 23rd row? (grid width $=10$ )' - was designed to probe participants' understanding of this property explicitly. The prior research summarised above suggests that students would have difficulty solving this question without appealing to an algorithm: they would not simply use the 'every nth' property of multiples. However, ten out of the fifteen participants who were asked this question effectively used the 'constant spread' property of multiples. Kori provides an example:

[...] because the grid width is 10 , it's not possible to skip, it's not possible to skip a row because it's only, there are only 7, each multiple of 7 is only 7 spaces away from the previous one. So it can't skip an entire row which is 10 spaces, because within those 10 spaces a multiple of 7 will occur.

Based on the participants' success with this problem, we propose that the visually-based 'constant spread' property of multiples is more effective in problem-solving contexts for students than the equivalent 'every nth' property.

It may appear puzzling that the 'every nth' property is problematic for students. The subtlety of this property is revealed by a comment made by Sandy in response to the last interview question, asking whether she had any surprising insights while working with the microworld. She had 
been surprised to discover that "The greater the number [generating the multiples] the greater the spacing is between the multiples, but the spacing between the multiples does not change and will remain the same number of spaces even though the number changes". Sandy knew that the multiples of a number $m$ will increase when $m$ increases but she was surprised to find, while working with the microworld, that no matter how large $m$ becomes, the space in between each pair of subsequent multiples will stay the same.

The visual representation of multiples highlights their 'constant spread' property, that is, that there is an equal number of cells between each multiple and the next. Student understanding of 'constant spread' does not follow from an additive understanding of multiples, as both Sandy's comment and prior research show. Perhaps the visual representation helps students move from an additive understanding of multiples to one which features the 'every nth' property? That is precisely what Michelle's comment suggests: "multiples follow a pattern in that every 3rd number is highlighted if we want multiples of 3 , it gives us an actual image, not just words to describe it".

\section{Multiples and Factors}

Prior research has noted that students have a tendency to characterise arithmetic sequences of non-multiples, that is multiples shifted, as being "sporadic" whereas sequences of multiples are described as "orderly". In the written assignments as well as the interviews, the participants in this study consistently and overwhelmingly characterised all arithmetic sequences (whether of multiples or non-multiples) using rich adjectives such as: "constant", "equally spaced", "continuously repeating", "distinctively patterned", "continuous", "sequential", "repeating", "predictable", "extending infinitely", "uniform", "regulated", "symmetrical", "systematic" and "orderly". In contrast, the participants characterised factors as being: "scattered", "sporadic", "non-patterned", "inconsistent", "not symmetrical", "random", "chaotic", "limited", "discordant", and "unorganised".

It could be that by working with multiples and factors at the same time - and having access to visual representations of both - the participants were able to see that sequences of multiples are more like arithmetic sequences of non-multiples, that is, multiples shifted, than they are like factors. In most classroom settings, without such strongly contrasting evidence, students might focus more on the differences between sequences of multiples and arithmetic sequences of non-multiples than on their similarities. 
In addition to the contrasting adjectives used for factors, the participants also noticed some distinctive visual characteristics of factors: "clustered near top", "bunched", "steadily decreasing" (as one moves towards the number to be factored from its factors), "finite", "contained", and "featuring big spaces". Carrie's description typifies that of the large majority of participants:

The visual image I have of factors are contained and finite. Visually they are clustered towards the top of the grid and thin out as the factors get larger. The multiple is visually separate from the factors and stands on its own.

When asked about any new or surprising discoveries he had made while working with the microworld, Andrew explained that he had never realised how, with factors:

Spaces between the original numbers and the next cell will be very large but as we move down to the next highlighted cell the space between it and the previous will be smaller and this pattern will continue until we reach one.

Though Andrew claims he "knew" this before, it seems that the microworld brought alive his knowledge of this important property, which is rarely made explicit in classroom teaching.

Several participants - without being asked - also mentioned that the first factor of a number $n$ would never be found more than halfway between 1 and $n$ itself; for example (Amber):

The visual image I have of factors is that there isn't a common difference. A factor of a number is never larger than half the number being factored, not including the number itself.

The visual representations of multiples and factors offered in the microworld seemed to have been adopted by many of the participants, at least in the course of the study. Some participants clearly displaced their previous visual representations - if they had any - with the grid-based ones, as Adam's description shows: "well after looking at this, I can see the multiples of 5 now on the grid, but before that I wouldn't have thought of that, like I probably would've thought of the number line instead". Amy pointed to one of the advantages of the two-dimensional representation: "this gave me an overall like look of numbers and how they interact almost with each other".

There was also evidence of a few participants retaining previous representations of factor trees and number lines. Interestingly, some participants managed to combine representations. For example, Diane seems to construct an analogy for multiples that is at least partly inspired by the microworld's visual representations: 
Multiples work together creating a pattern of some sort, such as a collection of columns, a checkerboard, a diagonal or collection of connected and disconnected diagonals. They spread far and wide, and keep on going forever, but are still somehow connected. Multiples make me think of emails which are written by one person, starting out at that one point and then keep getting forwarded and sent on and on ...

The participants in this study have constructed very distinct visual images of factors and multiples, as evidenced in the rich vocabulary of adjectives mentioned at the beginning of the section. In addition, Lisa explains how her own visual distinctions helped her overcome previous confusions with the words 'factors' and 'multiples':

I usually, before I got multiples and factors really mixed up, I couldn't remember which is which and now I know what factors look like, and what multiples would look like, so I just have a better understanding of the concepts.

Lisa may have previously been aware of the conceptual differences between factors and multiple, but now she seems to be able to use and apply the words correctly because of her immediately accessible visual images. (This ability to correctly and automatically use and apply vocabulary is perhaps an underrated challenge that students face in the mathematics classroom.) Finally, Kori points to the novelty of the representations offered:

I didn't really realise the patterns of multiples until it was showing visually in front of me, I knew they went on but I didn't, the difference between the multiples and the factors and the patterns that they created, I didn't really know that before. I never thought of it before.

The distinct visual images constructed by the participants also seemed to help them work with factors and multiples simultaneously, as exemplified by Christine's comment:

When we were told to create a grid of stripes, this was where the factors came into play. Although we were still using the method of showing multiples, the numbers we choose were specific numbers this time; they were factors of the grid width.

We have focused in this section on the effects of visualisation on participants' understanding and use of specific elementary number theory concepts. After a parallel discussion on the effects of experimentation we will return to the visualisation theme, and look more at the effects of visualisation on the affective and aesthetic dimensions of participants' mathematical experiences with Number Worlds.

\section{Effects of Experimentation Allowed within Number Worlds}

In this section, we examine the effects of experimentation on the participants' understanding of several concepts and their approaches to problem 
solving. First, we describe the range of experimental approaches we observed, both in the lab and during the interviews. We then focus our discussion on the way experimentation enabled students to encounter two important ideas: the effect that increasing a number has on its total number of factors, and the fact that the product of two prime numbers produces a non-prime number.

\section{The Range of Experimental Approaches to Problem Solving}

We use the term 'experimental approaches' to describe ways of discovering results that rely on empirical methods. Many of the assignment tasks encouraged the use of empirical methods by asking first for many examples of a certain phenomenon, and then for a generalisation. For instance, while working on Task 1, the participants we observed in the lab first found three examples of numbers having exactly three factors. Once they had gathered the examples, they then attempted to identify a pattern (all examples are square numbers) and make a generalisation. This experimental approach differs from a more deductive one, which a few students seemed to use in their assignment write-ups, ${ }^{3}$ which may have involved appealing to the Fundamental Theorem of Arithmetic.

Every participant that we observed in the lab relied heavily on experimental approaches. However, the situation was more varied and complex in the interviews. In contrast to the assignment questions, the interview questions were not structured to invite experimental approaches, primarily because they asked for predictions rather than patterns. Nonetheless, we gave the participants access to Number Worlds during the interviews in order to determine whether they would (and could) use it to help them solve problems. The range of use varied from not at all to complete reliance. Those who did rely on experimental methods seem to do so for different purposes, each of which corresponded to a component of the methodology of 'experimental mathematicians' identified by Bailey, Borwein and Girghenson (2003). We identified four of these distinct purposes: verifying analytically-derived results; discovering new patterns and relationships; testing and falsifying conjectures; gaining insight and intuition.

A few participants in the interviews - the 'anti-experimenters' - refused to use the microworld at all, preferring to rely entirely on non-empirical methods. One participant suggested that using Number Worlds to answer the interview questions was "cheating" while another just felt "more comfortable" using pencil-and-paper methods.

Among the 'experimenters', a few participants used Number Worlds for verification, to check results they had derived non-empirically. These 
participants would reason through a problem without using the microworld and then once they had a solution, try it with Number Worlds. It appears that these participants had developed a certain reliance on - and perhaps trust in - the visual feedback of Number Worlds. Since the interviewer was present to give feedback on whether or not their solutions were right, these participants knew that the visual feedback might provide additional guidance in case their solution was incorrect - something that the interviewer might not necessarily provide.

The majority of the participants we interviewed relied more significantly on Number Worlds during the interview than did the 'verifiers'. One subset of this majority, which we call the 'guessers' group, used the microworld to look for patterns and relationships in a way that was not guided by explicit conjectures or hypotheses. For example, one participant simply switched the 'four 'and the 'two' in his first attempt for Question 3 (see Appendix 3), without having any motivation he could articulate for doing so. While we judge this as undesirable behaviour, the participant was at least able to use the feedback to discern the effect of his actions and thus make a more considered guess - leading him, eventually, to use the microworld as did the subset we describe next. This subset, which we call the 'conjecturers' group, used the microworld to test and falsify conjectures. For example, while working on Question 3, one participant began by reasoning that the values of the multiples would have to remain the same. She then decided to investigate what the effect of changing the value of the shift would be. By observing the effect of several different values she was able to determine empirically, and then verify, the solution. The third subset, which we call the 'intuiters', used the microworld to help them think about a problem. For example, in trying to determine what kind of values produces the third image in Question 6 (see Figure 7), one participant decided to show the prime numbers, not in order to verify his conjecture, but in order to get a sense of what prime numbers look like on the grid. He knew that the image did not necessarily start at 1 , and was thus aware that Number Worlds might not give him the answer. Instead, he knew that he could use the visual patterns displayed by the microworld to gain insight.

This range of experimental uses of the microworld certainly reflects some learning style preferences, as well as participants' comfort level with computers. It would also seem that, among the heavy experimenters, 'conjecturers' display more mathematical sophistication, as they are able (or willing) to make more purposive guesses. In contrast, the 'intuiters' a group we had not expected to find - seem to display more sophistication vis-à-vis the microworld itself, knowing how to use it in more flexible 
ways. Similar characterisations of student engagement in a computerbased environment (involving learning limits with a computer algebra system) are described by Artigue (2002). This may suggest that the experimental uses described here are neither tool- nor content-specific.

As mentioned before, the range of experimental uses was most evident in the interviews. During the lab sessions, the participants we observed tended to rely more uniformly on experimental approaches - influenced most likely by the nature of the questions. In the following sections, we consider two specific concepts that such experimental approaches enabled the participants to encounter.

\section{'More of A, More of B'}

Prior research has discussed a belief found among students that large numbers have more factors than small numbers (Zazkis, 1999). This has been described as belonging to the family of intuitive beliefs "the more of A, the more of B", discussed in detail by Stavy and Tirosh (2000). These researchers show that such intuitive beliefs can act both as beneficial and misleading heuristics in scientific and mathematical problem solving, and it appears that experimentation in Number Worlds helped the participants directly confront their misleading belief in relation to number of factors.

Participants were given a set of exploratory questions to investigate before they began work on their written assignments. These were designed, in part, to familiarise the participants with Number Worlds' functionalities. One question asked the participants to find a number smaller than 45 that has more factors than the number 45 . During our observations in the lab, several groups of participants expressed surprise upon finding that numbers such as 30 and 24 , which are smaller than 45 , manage to have more factors. Naturally, we could have asked the same questions in a noncomputer environment, but the availability of experimentation allowed the participants to use a guess-and-check approach, and to focus on the results rather than on the onerous process (for some) of finding the numbers' factors. Many groups ended up showing the factors of most of the numbers smaller than 45 , a task that would have almost certainly been too tiresome without the microworld.

While neither the interviews nor the written assignments contained explicit questions about this belief, several participants made mention of it, perhaps recalling their experiences with the exploratory questions. Laura attributed her new understanding to the visual representations afforded by the microworld, probably because her comment was in response to a question about the visual images of concepts: "The visual image also helped me to better understand that just because a number is bigger, that 
doesn't mean it has more factors". However it is difficult to see how the visual image per se contributed to the development of her understanding; it is more likely that Laura's interaction with the visual image through experimentation helped her.

Kelly provides another explanation for the role of the experimental aspect of the microworld in helping her understanding of factors:

However, the number of factors for each number depends on several aspects. Looking at only a few numbers it is difficult to show such patterns; therefore, I have learned that is important to look deeper and find more proof to reasoning. By finding similarities between several small numbers with four factors we were able to make generalisations about all numbers with four factors.

By experimenting with many different examples, Kelly shows that it is possible to locate patterns which can displace intuitively acquired ones such as "more of A, more of B". A few students seemed in the midst of letting go of their intuitively guided conceptions. For example, Blake writes that: "In addition, in most cases there are fewer factors if the number is low, and more factors as the number gets higher, generally. This is not always true however as there are many large numbers that have few factors or are prime".

\section{Primes and Factors}

Previous research has shown that often students do not immediately recognise a number represented as a product as a composite number. Also, they do not recognise that a product of two prime numbers cannot itself be a prime number. During our interviews, participants were asked whether the prime numbers were closed under multiplication. Every single participant unequivocally provided the correct answer. When asked to explain why, the majority selected the Prime World in Number Worlds and chose two numbers to multiply - quickly finding the required counter-example. In his interview, Dale explains how this understanding was influenced by the microworld:

going into it I did have the information at hand on how we deduce the amount of factors a certain number has, but to be able to work with the applet and that showed me a different way. I mean, I realised maybe more so that uh two prime numbers form a composite, and stuff like that.

Dale's comment alludes to the fact that he did have the "information" required - the property concerning factors that was presented in class - but perhaps he gained conviction or immediacy by seeing several examples of two primes multiplying to produce a composite number, and also, seeing several examples of showing factors of prime numbers. In the following 
excerpt, Donald also attributes his new understanding to his work with the microworld, and particularly to the first task in the assignment:

This program has shown me visually that the product of two prime numbers, three and five for example, elicits a number with four factors: one, three, five, and the composite number fifteen.

One task asked participants to find general ways of producing numbers with exactly 3, 4 and 5 factors. Although, once again, they had encountered algorithms for doing so in class, many were surprised, as we observed in the lab, to find that the product of any two prime numbers produces a number with four factors. It would seem that the first task, in and of itself, could support the development of the participants' understanding that a product of two prime numbers cannot itself be a prime number. By asking students to identify a general way of producing a number with four factors, and the experimental environment in which to explore this, the participants were led to consider the product of prime numbers and to deal with many examples of products of primes yielding numbers with several factors. The microworld thus provided the vehicle through which we were able to set a task of a kind that we may not have been able to set in a non-computational environment.

\section{Students' Experiences Working with Number Worlds}

As the participants began working with Number Worlds in the lab, they seemed engaged and explorative. Many responded with surprise and even delight upon creating their first patterns of stripes and diagonals. There was, however, a small minority of students who simply objected to using the computer, finding it distracting or uncomfortable.

Both in their written assignments and during their interviews, the students had opportunities to describe qualitatively their experiences working with Number Worlds. They were explicitly invited to do so in the Final Task of the assignment and in the last interview question. A few noteworthy themes emerged: the aesthetic appeal of the patterns, the importance of connectivities, and the visual/experimental dimension of their mathematical explorations. Interestingly, these themes have a strong overlap with Burton's (1999) epistemological model that describes the way in which mathematicians come to know mathematics.

\section{Aesthetics Theme}

Many of the participants, both in the lab and during the interviews, commented on the aesthetic appeal of the diagonal patterns produced by certain inputs to 'show multiples of'. Whereas stripes were deemed "boring" by many, diagonals were evocative of patterns and relationships while possessing a pleasing degree of complexity. Nick explained 
that stripes are to be expected, but that diagonals "really mean there's a pattern". However, a few participants, like Mary, expressed most interest in stripes:

Multiples can create some visually pleasing stripes and diagonals along with some eyestraining checkerboards. There is a sense of order in the universe upon viewing some stripes on the grid. I look at those stripes and think that I can instantly recognise this regular pattern as a logical pattern.

While Mary finds that the stripes convey a "sense of order", Amy's experience is more emotional: "The repeating, infinite pattern is calming. I can see with my eyes open the pattern on the grid right in front of me. They all seem to be linked together, no matter how far away from the next multiple they are, they are all connected to each other". Other participants spoke of the patterns being "aesthetically pleasing", perhaps in part because of their constancy and predictability: "all the visual images [...] are constant and predictable and they are aesthetically pleasing in their design or pattern".

For some, the supposed beauty and elegance of number theory may have been manifested in the pleasing patterns they could create and manipulate on the grid. More importantly, the general appeal of the patterns seemed to have encouraged most participants to simply 'play around' and explore, as Lisa explained.

It was fun, just playing around with different numbers, seeing the patterns you could make, like often times I wouldn't look at the numbers, I would look at rows and look at patterns, and how those corresponded to each other and then put the numbers in afterwards, so experimenting around with that, I could figure out different ways of getting the things without necessarily having to use numbers.

The participants' aesthetic response to the microworld's patterns seemed to give them access to greater expressive power, allowing them to communicate both their ideas and feelings through Number Worlds. Their aesthetic connection went beyond a frivolous attraction to patterns: it provided them with a new, qualitative way of thinking about numbers and their relationships. This kind of connection has to do with the process of mathematical thinking rather than its product, the latter being the arena in which the aesthetic plays the role of distinguishing good from not-so-good mathematics.

\section{Connectivities Theme}

Many of the participants talked about the way the microworld allowed them to see relationships, and to see how different concepts connected. Connectivities could be between different concepts, as it was for Lisa, who said that Number Worlds "helped me understand things better, [...] it just kind of connects different parts of multiples and math and stuff like 
that altogether". Connectivities could also be between different types of understanding, as it was for Jake, who described the connections facilitated by the microworld as a "springboard" to help his understanding. Connectivities could also simply be within the objects and operations of the microworld. For example, Jessica talked about the way the visual display emphasised connections between the grid's numbers: "I don't know, it was neat to see for the factors, the patterns you can make with all the different ways, like checkerboards and all that kind of stuff, it was neat that numbers can actually do that when you put them all together like that". Katherine emphasised the way in which the visual image helped forge connections for her:

I find it interesting, the whole idea of the cells highlighting and seeing how they relate and just like even looking at these pictures too, kind of making everything back to the visual image that it created and how they related to each other and how it's like not prime or how it is prime, because of it.

Carrie explained how the microworld emphasised relationships for her because it got her "into a different way of thinking about it, like it's their relationship, it's numbers and their relationship to each other that I found interesting, visually". She also explained that these relationships were more clear on the Number Worlds grid than on a number line: "if you do a number line, every even number is, you see it in a line, but somehow in a pattern you really see just the relationship to each other".

Finally, connectivities could be between mathematics and the outside world, as it was for Vanessa, who talked about how her Number Worlds experience has changed the way she thinks about numbers in her everyday life. Based on Devlin's (2000) characterisation, her statement bears striking resemblance to the way mathematicians relate to mathematical objects: "mathematicians think about mathematical objects using the same mental faculties that the majority of people use to think about other people" (p. 262). For mathematicians, numbers are like "characters in the mathematical soap opera": but, instead of wondering about marriages and love affairs, mathematicians wonder about mathematical facts and relationships. How is this number related to that one? What kinds of number have a certain property?

I will definitely take what I've learned about manipulation of numbers and seeing patterns with me as I use numbers in other contexts of my life [...] I definitely learned a lot of different ways of looking at numbers, I don't just, like I'm looking at the number 64 right now, before I was just kind of taking advantage of, of it being 64, but now I kind of think how is that related to the other numbers that are in the set of numbers that I'm looking at.

Like Vanessa, many participants talked about the way in which the microworld gave them a "different way of looking at numbers". For some this different way was sufficiently novel to provoke an emotional reaction 
of curiosity and engagement. And for some, the different way provided access to different ideas and relationships that could be connected to previous ones.

\section{Visual/Experimental Theme}

The third theme has to do with the possibilities of interaction and feedback offered by the microworld; the participants mentioned three distinctive opportunities this gave them: working with ideas, testing theories, and working with many examples. Katherine spoke of the differences between classroom learning and her experience with Number Worlds in terms of interactions and the ability to work with ideas:

usually when you're in class the professor would be saying okay, well there's this prime number, and this is how they relate and that kind of stuff, but then once you see it on paper, as opposed to like once you're interacting with it, it makes a difference because you can kind of see how they relate a bit more.

Amber also spoke about the importance of experimenting and making predictions, in contrast to just being told about a certain property or relationship; she stresses how being able to work with the ideas gives her a sense of agency:

when you're actually working with it and it's not somebody just telling you how it is and you're actually seeing it for yourself, so if you get to try things out and you get to see what your results are and you get to make predictions and then see if they're right or wrong, I think that helps. Because it's not so much somebody tells you something.

For Jake, the ability to not only test theories but also, to test theories quickly was important: "The program allowed me to test potential theories as they entered the mind, and quickly enough so that the thought was not lost; the program did the time-consuming work". Jake's comments captures the way in which the microworld may support qualitative reasoning, allowing students to test ideas that are not fully formed. Kyle commented on how the ease of experimentation helped make concrete his ideas-in-formation: "being able to change variables and viewing the outcomes can clarify and solidify ideas which are more often than not just floating around in a person's head". Since it was easy to perform any operation, Katherine found she was less concerned about making mistakes, and more inclined to just try things out: "you could just quickly click and say this, and then reset it and try something different if it didn't work, it was pretty quick that way you could just do it and the picture was there in front of you, right or wrong, okay it's wrong, so let's just erase it and try something new". The ease with which participants could experiment with a wide range of numbers may have helped them move beyond the kind of pattern spotting that frequently characterises the approaches of students 
- where a few examples using small numbers are seen as sufficient to determine a rule or pattern.

Kori claimed that the computer's ability to do most of the computations enabled her to reflect on why things were happening: "it shows it to you instantly, so you can maybe think more about what's going on behind it, rather than having to actually do the calculations, you can think about why it's working". Although some educators have worried that the ease of experimentation will lead students to mindless trial-and-error interactions, Kelly claimed that she learned about the importance of looking beyond a few examples: "Looking at only a few numbers is difficult to show such patterns; therefore, I have learned that is important to look deeper and find more proof to reasoning".

The participants quoted above articulate the learning benefits offered by the computer's power to provide feedback and interactive experiences. Together, they suggest that Number Worlds facilitated their explorations of elementary number theory concepts and problems.

\section{CONCLUSIONS}

In this article we have outlined the design features of the Number Worlds microworld and described the interaction of a group of pre-service elementary teachers with this microworld. Prior research has repeatedly shown that the concepts and relationships underlying elementary number theory are elusive and fleeting for students. In comparing the work of participants in this study with that research, we find that interaction with Number Worlds had a positive effect in helping participants construct these concepts. In particular, the previously reported confusion between factors and multiples, belief in larger numbers having more factors, and the lack of recognising the 'every nth' property of multiples were either absent or infrequent among the participants working with Number Worlds. Furthermore, we observed stronger understanding of factors and multiples, especially in relation to their distribution within the natural numbers. Based on observations in the computer lab, students' written assignments and clinical interviews, we attribute this effect to the two major factors we had hypothesised would be pivotal: (1) the novel visual representation inherent in Number Worlds and (2) the possibility of experimentation.

The visual presentation of the natural numbers within Number Worlds offers an alternate representation of the numbers compared to that of the more traditional number line. This alternate representation accentuates the patterns inherent in arithmetic sequences of multiples, as well as arithmetic sequences of non-multiples generated by 'shifting' the multiples by an 
integer value. It also allows users to see patterns, or lack thereof, in the structure of factors, square numbers, and primes. The second feature influencing the participants' understanding of basic elementary number theory relationships lies in the ease with which the microworld accommodates experimentation. Participants were able to interact with Number Worlds in a way that allowed them the freedom to think both about the microworld and with it.

These two features - visual representation and experimentation - are in fact closely interrelated. The crucial link between the two is the attention to patterns. Number patterns presented in a novel visual manner provoked aesthetic responses that invited experimentation, which generated new patterns, thereby reinforcing aesthetic engagement. Furthermore, the regularity of visual patterns led students to make conjectures, which could be in turn tested through experimentation and visual feedback. The participants' conjectures show their perceptions of the relationships among the concepts of number theory, and the depiction of these concepts in the microworld. This cycle of experimenting-observing-conjecturingexperimenting ultimately aids learners to construct their understanding of underlying concepts or to strengthen it through an added perspective.

\section{NOTES}

1 The Number Worlds microworld is written in Java and is available on-line as an applet at http://hydra.educ.queensu.ca/java/NumberWorlds/.

2 This approach was inspired by Brown (1978), who used similar ways of re-examining ideas in elementary number theory by shifting the focus from natural numbers to other domains.

3 It is not clear from the assignments whether these participants actually used experimental approaches but stated their results - as most mathematicians do - without mention of them, or whether they in fact relied solely on non-empirical methods.

\section{APPENDIX 1}

Tasks to explore in Number Worlds

1. Can you highlight the even numbers without using the Show Evens button? Can you highlight the odd numbers?

2. Set your grid width to 10 . Show multiples of 10 . What could you do to highlight all the numbers in the third column?

3. Can you find a number smaller than 45 that has more factors than the number 45 ?

4. Can you get the numbers $7,14,21, \ldots$ highlighted? Can you get the numbers 8,15 , $22, \ldots$ highlighted? 
5. Change to the Odd World. Try adding two numbers. What do you notice? Try multiplying two numbers. What do you notice? Do the same thing in the Even and Prime worlds.

6. What could you do to highlight the number 169 (without clicking it directly!)? How about the number 320 ? Use the Increase by one row button to check your answer.

7. Can you get all the numbers in the first row of the grid highlighted? (Hint: you can change the grid width.)

8. Choose Show Primes in the Natural World. Now go to the Odd World and choose Show Primes. What do you observe about the location of the highlighted numbers?

\section{APPENDIX 2}

Written Response Tasks

\section{Task 1. Focus on Factors}

1. Can you give three examples of numbers that have exactly 2 factors?

Can you give three examples of numbers that have exactly 3 factors?

Can you give three examples of numbers that have exactly 4 factors?

Can you give three examples of numbers that have exactly 5 factors? Check your examples with Number Worlds.

2. Can you find a number greater than 500 that has exactly 4 factors? Describe how you found this number and explain how you could find another such number.

Can you find a number greater than 500 that has exactly 5 factors? Describe how you found this number and explain how you could find another such number.

\section{Task 2. Stripes, Checkerboards and Diagonals}

1. Describe at least three different ways for creating a grid of stripes, working with different grid widths. (Two examples of a "grid of stripes" are shown below.) Describe a general procedure for creating a grid of stripes.
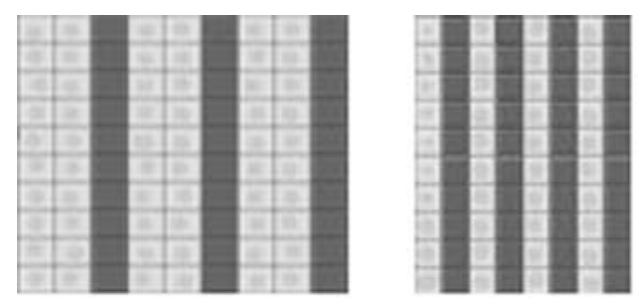

2. Describe at least three different ways for creating a checkerboard grid. Describe a general procedure for creating a checkerboard grid. Is it possible to create a checkerboard for any grid width?

3. Describe at least three different ways for creating a grid of diagonals (either left to right or right to left). Describe a general procedure for creating a grid of diagonals.

4. Describe at least three different ways for creating a grid of "disconnected diagonals", as shown in the figure below. Describe a general procedure for creating a grid of disconnected diagonals. 


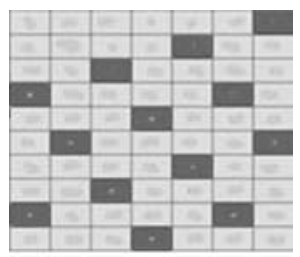

5. What do all the visual images you created in a) through d) have in common?

\section{Task 3. Prime Distributions}

1. In the Natural World, set your grid width to 4. Choose Show Primes. What do you notice about the highlighted numbers? Explain.

2. Now set your grid width to 6 . Choose Show Primes again. What do you notice about the highlighted numbers? Explain.

\section{Task 4. Multiples}

1. Using Number Worlds with a grid width of 10, someone has chosen to show the multiples of $k$. If there are three cells highlighted, what are the possible values of $k$ ? Explain your answer.

2. How would the answer in part (a) change if more than three cells were highlighted? Explain.

3. How would the answer in part (a) change if the grid width was different than 10 ?

\section{Final Task}

Write a paragraph that describes the visual image you have of multiples and the visual image you have of factors.

\section{APPENDIX 3}

\section{Interview Questions}

1. Here we are looking at the multiples of 7 , with a shift of 2 . The grid width is 7 . What would happen if we change the shift to 5 ? What would happen if we change the multiples to 8 (Returning to multiples 7, shift 2) How could we get 2 highlighted?

2. The grid width is 10 , with multiples of 4 , shift 2 highlighted. Can you create a grid of stripes by changing only one thing (either grid width, multiples or shift)?

3. The grid width is 9 , with multiples of 4 , shift 2 highlighted. The first number in the grid is 190 . How else could we highlight the same numbers?

4. Show how you could verify the following statements using Number Worlds: a) the set of even numbers is closed under addition; $b$ ) the set of odd numbers is closed under multiplication; c) the set of prime numbers is closed under multiplication

5. Is there a multiple of 7 in the 9th row of your grid? What about the 23rd row? 
6. Consider each picture. Is it a picture of factors, multiples, primes or squares? How do you decide?
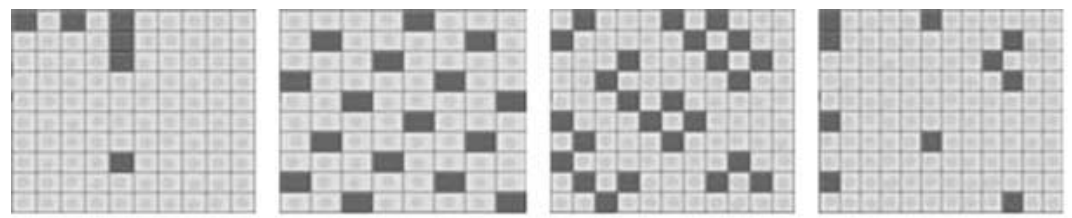

7. General invitation for reflection: What have you found surprising or helpful or interesting in your experience with Number Worlds?

\section{REFERENCES}

Artigue, M. (2002). Learning mathematics in a CAS environment: The genesis of a reflection about instrumentation and the dialectics between technical and conceptual work. International Journal of Computers for Mathematical Learning 7(3): 245-274.

Bailey, D., Borwein, J. and Girgensohn, R. (to appear). Experimental Mathematics: Computational Paths to Discovery. Boston, MA: AK Peters.

Brown, S. (1978). Some "Prime" Comparisons. Reston, Virginia: National Council of Teachers of Mathematics.

Brown, A., Thomas, K. and Tolias, G. (2002). Conceptions of divisibility: Success and understanding. In S.R. Campbell and R. Zazkis (Eds), Learning and Teaching Number Theory: Research in Cognition and Instruction (pp. 41-82). Westport, CT: Ablex.

Burton, L. (1999). The practices of mathematicians: What do they tell us about coming to know mathematics? Educational Studies in Mathematics 37(2): 121-143.

Devlin, K. (2000). The Math Gene: How Mathematical Thinking Evolved and Why Numbers Are Like Gossip. New York: Basic Books.

Edwards, L. (1995). Microworlds as representations. In A.A. diSessa, C. Hoyles and R. Noss (Eds), Computers and Exploratory Learning (pp. 127-154). Berlin/Heidelberg: Springer-Verlag.

Eisenberg, T. and Dreyfus, T. (1991). On the reluctance to visualize in mathematics. In W. Zimmerman and S. Cunningham (Eds), Visualization in Teaching and Learning Mathematics (Vol. MAA Notes Series, pp. 25-37). Washington, DC: MAA Press.

Ferrari, P. (2002). Understanding elementary number theory at the undergraduate level: A semiotic approach. In S. Campbell and R. Zazkis (Eds), Learning and Teaching Number Theory: Research in Cognition and Instruction (pp. 97-116). Westport, CT: Ablex Publishing.

Goldenberg, P. (1989). Seeing beauty in mathematics: Using fractal geometry to build a spirit of mathematical inquiry. Journal of Mathematical Behavior 8: 169-204.

National Council of Teachers of Mathematics (1989). Curriculum and Evaluation Standards for School Mathematics. Reston, VA: NCTM.

Noss, R. and Hoyles, C. (1996). Windows on Mathematical Meanings. Dordrecht, The Netherlands: Kluwer Academic Publishers.

Noss, R., Healy, L. and Hoyles, C. (1997). The construction of mathematical meanings: Connecting the visual with the symbolic. Educational Studies in Mathematics 33: $203-$ 233. 
Stavy, R. and Tirosh, D. (2000). How Students (Mis-)Understand Science and Mathematics: Ntuitive Rules. New York: Teachers College Press.

Zazkis, R. (2000). Factors, divisors and multiples: Exploring the web of students' connections. Research in Collegiate Mathematics Education 4: 210-238.

Zazkis, R. (1999). Intuitive rules in number theory: Example of "the more of A, the more of B" rule implementation. Educational Studies in Mathematics 40(2): 197-209.

Zazkis, R. and Campbell, S.R. (1996a). Divisibility and multiplicative structure of natural numbers: Preservice teachers' understanding. Journal for Research in Mathematics Education 27(5): 540-563.

Zazkis, R. and Campbell, S.R. (1996b). Prime decomposition: Understanding uniqueness. Journal of Mathematical Behavior 15(2): 207-218.

Zazkis, R. and Liljedahl, P. (2002). Arithmetic sequence as a bridge among conceptual fields. Canadian Journal of Science, Mathematics and Technology Education 2(1): 93120.

Faculty of Education

8888 University Drive

Simon Fraser University

Burnaby, BC, V5A $1 S 6$

Canada

E-mail:nathsinc@math.msu.edu 
